\title{
A First Analysis of the Potential Impact of Climate Change on the Efficiency and Reliability of Solar and Hydro Energy Sources
}

\author{
B. A. Carreras \\ Depart. Fisica, \\ Universidad \\ Carlos III, \\ Madrid, Spain \\ bacarreras@gma \\ il.com
}

\author{
J. M. Reynolds \\ Barredo \\ Depart. Fisica, \\ Universidad \\ Carlos III, \\ Madrid, Spain \\ jmrb2002@gmai \\ 1.com
}

\author{
D. E. Newman \\ Physics Dept. \\ University of \\ Alaska \\ Fairbanks \\ denewman@alas \\ ka.edu
}

\author{
U. S. Bhatt \\ Dept. of \\ Atmospheric \\ Science \\ University of \\ Alaska \\ Fairbanks \\ usbhatt@alaska. \\ edu
}

\author{
D. Gomila \\ Instituto de \\ Física, IFISC \\ (CSIC-UIB). \\ Universitat de \\ les Illes Balears \\ damia@ifisc.uib- \\ csic.es
}

renewable energy resources as the secular change in the resource. These changes can impact the electric power system in several ways. On the load side, demand can change. For example, heat waves can increase demand and load variability. On the generation (supply) side, as discussed above, changes in weather and climate can impact the availability of solar, wind and hydro power (both positively and negatively). Finally, and very importantly, the increased variability in these can greatly increase the stress on the system $[11,12]$ and the need for increased energy storage capabilities $[13,14]$ in order to ensure that the supply can meet the demand when the resource is at its minimum and the load is at its maximum. It should be noted that some renewable energy resources can be more broadly distributed (i.e., not as concentrated in a very large plant) which can to some degree ameliorate the increased stress from the high variability $[11,12]$. As penetration of these renewable resources increases these effects become only more important. Global warming affects all possible renewable energy sources. Here we will examine the possible impact it has on two of these energy sources, solar and hydro (though we will also mention wind briefly). To do this in the preliminary analysis we look at two locations which are far apart both in distance and climate region. One is Palma de Mallorca in the Balearic Islands, the other is Cordova in Alaska. At each of these two locations we have about 60 years of meteorological data, which should be sufficient to detect any impact of global warming as during this period the average temperature of Earth [15] increased about $1{ }^{\circ} \mathrm{C}$ as shown in Figure 1.

As one motivation for this work, recent reviews point to several needs in this area. A review and synthesis of studies on the impacts of climate change on renewable energy shows that most research has focused on hydropower and wind energy and highlights the need 
for regional analysis that also addresses economic impacts [16]. The uncertainty of climate impacts on energy at regional and global scales from a review of the literature motivates the need for a coordinated effort to develop a consistent framework for such assessments [17] which drives this attempt to build such a framework.

Of importance to power production is the "amount" of a resource available. To investigate the potential impact of climate change on this we first examine any secular change in the resource. However, because renewable energy sources are already characterized by great variability, one of the concerns is the possible increase in this variability. To measure the variability we use multifractal analysis of the data, calculating the intermittency coefficient $\mathrm{C}(1)$ (as described in the next section) together with the impact that this variability has on the storage needed. The fundamental goal of this work is to allow exploration of the storage needs for various renewable electric power sources and how they might change given climate change. Additionally, the analysis seeks to understand the change in stress on the power transmission system and the change in risk inherent to that system. This paper demonstrates the preliminary steps towards this goal.

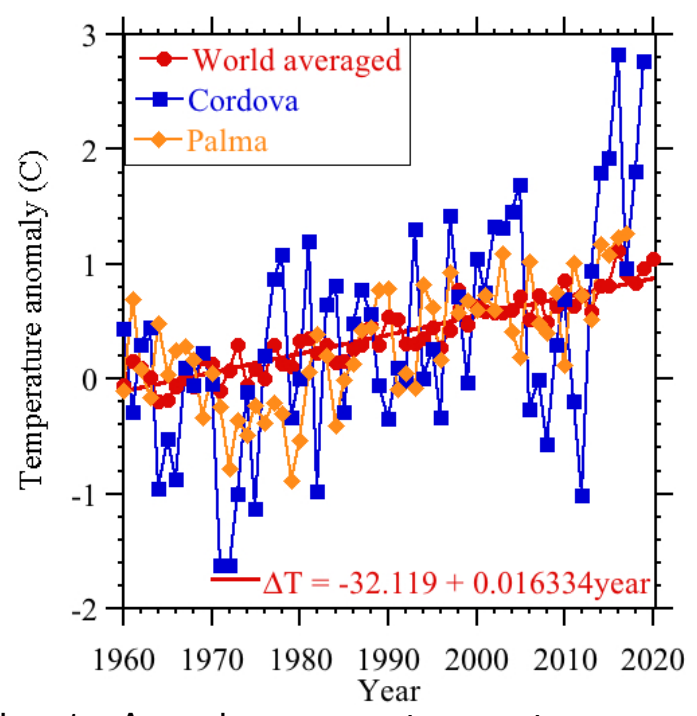

Fig. 1. Annual average temperature anomaly showing an increase in the average temperature of the Earth as well as the Cordova AK and Palma Spain sites over the time period considered in degrees Celsius.

In the next section we briefly present the analysis tools used in this study. In Section 3 we will discuss the meteorological data used and in sections 4 and 5 we discuss the results for Solar and hydro energy sources, respectively.

\section{Analysis tools}

In order to measure the impact of the climate change on the energy production of the renewable energies and to examine trends in the resource, we will measure the variability of the meteorological data to quantify how that impacts the needed energy storage. Here we describe some of the basic analysis tools that are used in this study.

\subsection{Intermittency}

To evaluate the variability of the meteorological data, we use multi-fractal analysis and calculate the intermittency of temporal signals. The method is described in Refs. [18, 19, 20]. Given a meteorological time series $X=\{x i, i=1, \ldots, N\}$ that has been sampled at a constant sampling rate, we calculate:

$$
\varepsilon(1, \mathrm{i})=\frac{\left(\mathrm{x}_{\mathrm{i}}-<\mathrm{x}_{\mathrm{i}}>\right)^{2}}{\left\langle\left(\mathrm{x}_{\mathrm{i}}-<\mathrm{x}_{\mathrm{i}}>\right)^{2}\right\rangle}, i=1, \ldots, N
$$

where

$$
\left\langle\mathrm{x}_{\mathrm{i}}\right\rangle=\left(\sum_{i=1}^{N} \mathrm{x}_{\mathrm{i}}\right) / N
$$

This measure can be averaged over sub-blocks of data of length $\mathrm{n}<\mathrm{N}$, as follows:

$$
\varepsilon(n, i)=\frac{1}{n} \sum_{j=0}^{n-1} \varepsilon(1, i+j)
$$

We then calculate the q-moments, $\left\langle\varepsilon(\mathrm{n}, \mathrm{i})^{\mathrm{q}}\right\rangle$. In a given range of n-values, these moments are expected to scale like [18]:

$$
\left\langle\varepsilon(n, i)^{q}\right\rangle \propto n^{-K(q)},
$$

where $\mathrm{K}(1) \equiv 0$. If the time series $\mathrm{X}$ is mono-fractal, the function $\mathrm{K}(\mathrm{q})$ is asymptotically linear in $\mathrm{q}$, otherwise the series is multi-fractal. We can now introduce the parameter $\mathrm{C}(\mathrm{q})$ which is defined as [21]: 


$$
C(q)=\frac{K(q)}{q-1}
$$

The parameter $\mathrm{C}(1)$ is the intermittency parameter. Due to the singularity at $\mathrm{q}=1$, the intermittency parameter must be calculated as:

$$
C(1)=\left.\frac{d K}{d q}\right|_{q=1}
$$

The value of C(1) goes from 0 , for a mono-fractal (low intermittency) time series, to 1 (high intermittency) as the multi-fractal character increases. The reason one uses this parameter is to quantify the level of multifractality (or intermittency) of the measured signals. For most of the data analyzed here the sampling rate is one day.

For precipitation data we included the intermittency sampled on both an hourly and daily basis. Other time scales could be of interest particularly for wind data but that will be presented elsewhere. When looking at the data we discuss the average rate of annual change obtained from the linear fit and the corresponding uncertainty determination. This linear fit should not be thought of as an actual functional description of the data (ie it need not be linear), but simply as a measure of the average rate of change over the time period considered.

\subsection{Calculating the storage needed}

An important component of the reliability of the power production which is impacted by the variability of the renewable energy is the energy storage. Here we calculate the energy storage needed in order to be able to guarantee an average power supply in the presence of the highly variable power production as described in reference [21]. First we need to evaluate the daily power flow that can be delivered to the customers in order to maximize efficiency and minimize the cost of storage. For example, if $\mathrm{P}(\mathrm{t})$ is the Solar power (or Wind power) produced every day and $\mathrm{P}_{\mathrm{F}}(\mathrm{t})$ is the power flow out of the plant, we can estimate the energy storage needed to ensure this power flow by calculating:

$$
R(t)=\int_{0}^{t}\left(P\left(t^{\prime}\right)-P_{F}\left(t^{\prime}\right)\right) d t^{\prime}
$$

The power flow out $P_{F}(t)$ is the one that minimizes the maximum of $\mathrm{R}$ with the condition $\mathrm{R}>0$ for all times. Then, maximum value of $\mathrm{R}(\mathrm{t})$ along the time interval of interest, MaxR, gives us the storage needed.
The result of this storage calculation is strongly affected by large events. Therefore, it conveys different information than the intermittency.

In this work we are trying to evaluate the effect of climate change, so we consider isolated electric power production facilities assuming they have the same characteristics in each location. If we were taking this one step further, we would include the power production plants in the corresponding grid and then the storage needs would change as would the optimal size of the plant. However, here we are not yet discussing the effects of and on the grid. We are also assuming a constant power production during each month. In a more realistic case we would optimize the production to the conditions of the grid and local climate. With these assumptions the needed storage is a good measure of the impact of climate, although it must be viewed as a relative measure in time and location.

\section{Data used in this study}

The basic historical reanalysis data that we have used is ERA5 and is obtained from the European Centre for Medium Range Weather Forecasting (ECMWF) Copernicus Climate Change Service [22]. Reanalysis combines observational data and gap-fills using a weather forecast model to construct a gridded data set of the atmosphere. From this source we have obtained hourly data from 1960 to 2020 for temperature, total surface solar radiation, precipitation amount and wind speed. We have combined the hourly data to construct daily data. It is on this daily time scale that we analyzed the data to model the generation power of the renewable energy [21].

The first characteristic that the data allows us to evaluate is the local impact of climate change on the two locations that we are considering. These results are shown in Fig. 1.

At both locations, we can see a consistent increase in the yearly averaged temperature. Also, in both cases the increase is similar to or slightly larger than the increase of the averaged global temperature. The slope of the global temperature data is $\sim 0.016{ }^{\circ} \mathrm{C} / \mathrm{yr}$ while the Palma data shows $\sim 0.020{ }^{\circ} \mathrm{C} / \mathrm{yr}$ and Cordova with $\sim 0.029^{\circ} \mathrm{C} / \mathrm{yr}$. This suggests that it should be possible to detect the impact of this warming on the local renewable sources. It's also worth noting that the rate of change is larger in Cordova then it is in Palma, consistent with the notion of polar amplification [23].

In Palma we can complement the ERA5 data with AEMET data [24] to test the consistency of the input data. 


\section{Impact on solar plants}

The first data set we analyze is the one relevant to solar power. The ERA5 has hourly data for total surface solar radiation that can be directly related to the solar power production. This includes both the direct and indirect solar radiation received and therefore available at the surface. Importantly, in this work we do not scale this to account for the efficiency of the conversion, i.e., we are not including any particular model for the solar power "plant". First, we measure the intermittency, $\mathrm{C}(1)$, of this data on a daily scale. We do not look at the hourly data for the solar due to the dominance of the diurnal cycle in that time series. In Fig. 2 we plot the result for the two locations.

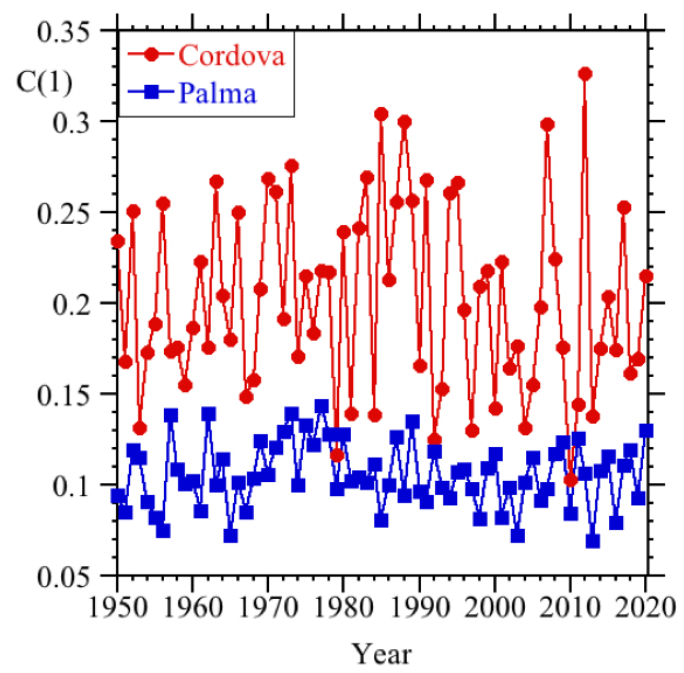

Fig. 2. Intermittency parameter for the solar radiation at the two locations.

Clearly the level of intermittency is much larger in Cordova (averaging $\sim 0.2$ ) than in Palma (averaging $\sim 0.1$ ). Additionally, the variance in the intermittency parameter is much larger in Cordova. The large fluctuations in the intermittency parameter makes it very difficult to assess the existence of a secular trend, perhaps a slow increase in time. If a trend does exist, it is very small and requires longer data sets measure reliably.

We can look in both cases at the storage needs for a plant. To do that, we group the data in increments of 5 years and based on the performance needs for the plant we calculate the storage required in each period. The results are shown in Fig. 3. We see that the storage needed for a power plant is larger in Cordova than in Palma. This is consistent with the high intermittency of the Cordova data. Additionally, we can see that both locations exhibit an increase of the storage need that is consistent with the secular trend likely due to global warming. Although was no clear increase in the case of the intermittency parameter, there is some correlation between the storage needs and the intermittency parameter.

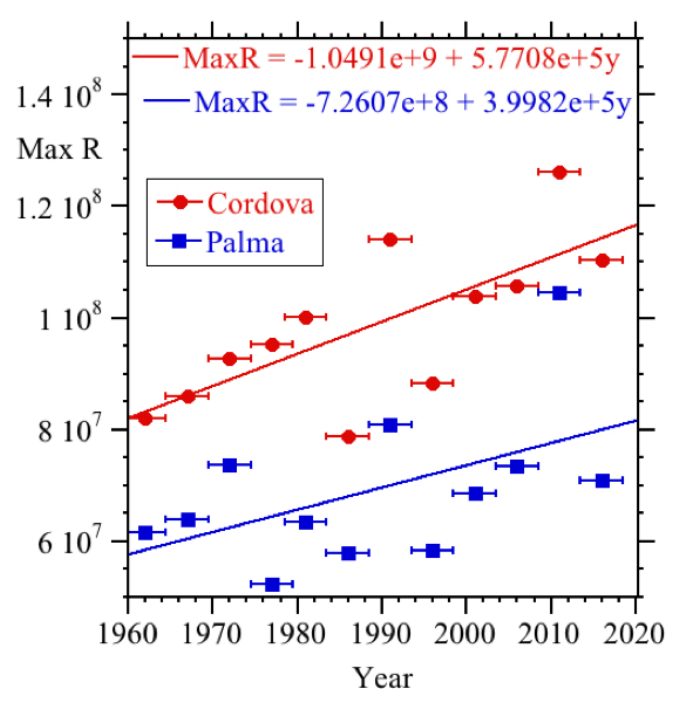

Fig. 3. Storage needed for a solar plant at the two locations.

Because of the secular changes due to climate change another relevant issue to be analyzed is the power production at the plant. These secular changes are changes in the average incident surface radiation due to changes in cloud cover, number of sunny days and cloud opacity. In Fig. 4 we show the averaged production, again over periods of five years at the two locations as a function of time.

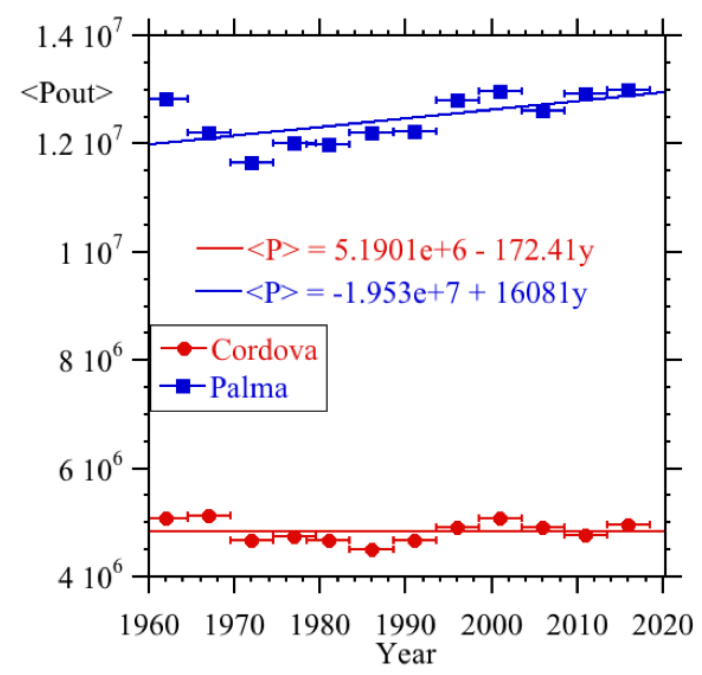

Fig. 4. Averaged power production at a solar plant on the two locations. 
We can first see that in Palma the production of solar power is higher than in Cordova. This is of course consistent with Cordova being a much cloudier location then Palma a well as the much larger intermittency in Cordova. Furthermore, the production in Palma clearly increases over time while for Cordova it remains practically flat. Looking in detail one finds in Cordova a small increase from 1970 to 2019 , but the optimal production in the 50's and 60's was high and that distorts the overall growth.

One possible measure of the efficiency of highly variable renewable energy power plants is the ratio of the energy storage needed, Max R, to the averaged energy production in one day, $<\mathrm{E}>$, that is $\mathrm{MaxR} /<\mathrm{E}>$. The lower this quantity is, the more "efficient" the system is in the sense that less energy storage is needed per power production unit. In Fig 5, we have plotted this quantity for both locations. It is clear from the figure that in Palma the solar energy can be a much more efficient system, as one could also conclude from the previous results. The very low efficiency of solar for Cordova is due to the large intermittency and high variability which makes it very costly from a storage perspective to get high reliability power from solar.



Fig. 5. Ratio of the energy storage needed to the averaged daily energy produced by a solar plant in Cordova and Palma.

\section{Impact on hydro plants}

Globally there is clear evidence of the impact of the climate change on precipitation. In some regions there is a significant increase in the intensity and intermittency of precipitation events $[6,7]$. There have already been evaluations of the impact of climate change on hydro production in some locations, like New Zealand [25]. In this work we will concentrate on the same two regions we have analyzed in the previous section.

We consider the precipitation data, which is the data relevant to hydro plants. Once again the ERA5 dataset has hourly data for precipitation for both locations that can be used to evaluate the power production. Again, we are not using a specific generation model used for the hydro power plants. Rather, we are using the available water from precipitation as a surrogate for the available power. First we measure the intermittency of this data on both hourly and daily time scales. In Fig. 6 and 7 we show the results for the two locations.

We can see once again that the fluctuations in the intermittency parameter can be large. This time the intermittency and variance in the Palma data is much larger, on the order of a factor of two for both the hourly and daily data. This is opposite of the intermittency result for solar radiation which makes sense because Cordova is a consistently rainy location with an average annual precipitation of $3,769 \mathrm{~mm}$ and with 125 rainy days/year while Palma averages only $402 \mathrm{~mm}$ of precipitation per year. These results however do have some secular behavior with Palma showing an increase in the intermittency in the hourly data while Cordova has a small decrease in the hourly data.

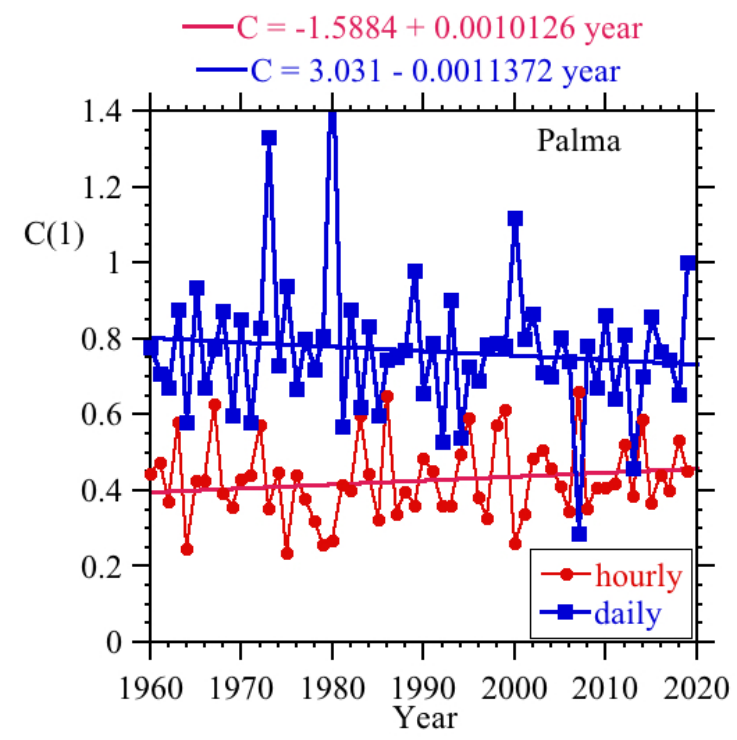

Fig 6. Intermittency parameter for the precipitation in Palma for hourly and daily data. 


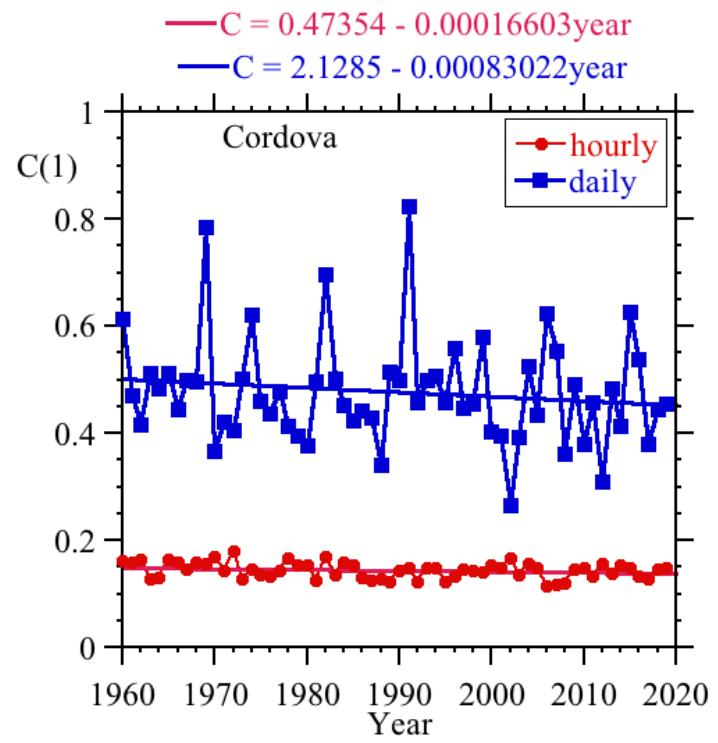

Fig 7. Intermittency parameter for the precipitation in Cordova for hourly and daily data.

To confirm these analyses in a location where climate change effects on precipitation are already being documented [26] we have done an analysis of the intermittency in a location in northern California, Lake Oroville, where we know the impact has been clear. In Fig 8, we show the intermittency parameter as a function of time calculated using ERA5 data for Lake Oroville.



Fig 8. Intermittency parameter for the precipitation in Lake Oroville

We see a consistent increase with time of the averaged intermittency, but more importantly the peaks of the intermittency increase at an even faster rate. These results for the Lake Oroville data are consistent with the increased drought periods and more extreme rainfall events. We therefore have clear evidence of different impacts on the intermittency as well as on secular trends in different regions. This validates the value of this type of analysis for power production.

It is worth repeating that when looking at the intermittency data we discuss the average rate of annual change obtained from the linear fit and the corresponding uncertainty determination. The data clearly has a great deal of variability, and this linear fit should not be thought of as an actual functional description of the data (i.e., it need not be linear), but simply as a measure of the average rate of change over the time period considered. The slopes and uncertainties are shown in Table 1. The main points to be gleaned from this table are: 1) the hourly data has less uncertainty in the slope calculation, 2) the Cordova intermittency is decreasing with time (ie has a negative slope) with the sign of the slope outside the error bars, particularly for the hourly data, 3 ) the Palma data shows an increasing intermittency in the hourly data (outside the error bars) but for the daily data both signs of the slope are inside the error bars, 4) the Oroville data has a increasing intermittency with once again the hourly data slope sign outside the error bars.

\begin{tabular}{|l|l|l|l|}
\hline $\begin{array}{l}\text { Time } \\
\text { scale }\end{array}$ & Slope & Uncertainty \\
\hline & Cordova & & \\
\hline Hourly & & -0.000166 & $9.013 \mathrm{e}-05$ \\
\hline Daily & & -0.000830 & 0.000746 \\
\hline & Palma & & \\
\hline Hourly & & 0.001013 & 0.000588 \\
\hline Daily & & -0.001137 & 0.001377 \\
\hline & Oroville & & \\
\hline Hourly & & 0.000771 & 0.000551 \\
\hline Daily & & 0.000971 & 0.001524 \\
\hline
\end{tabular}

Table 1 The Fitted linear slopes and the uncertainty in those slopes for the precipitation intermittency data.

Returning to the study locations of Cordoba and Palma, we examine the storage needs for hydro plants. Again, we bin the data in 5-year periods and based on the optimal performance needs for the plant we calculate the storage required in each period. The results are shown in Fig. 9. We can see that neither in Palma nor Cordova have any significant change in their storage needs over the time period. Particularly we can see that in both Palma and Cordova there is no significant increase in the storage needs in time. In the case of Palma, there a slight decrease. 




Fig.9. Storage needed for a hydro plant in Cordova and Palma.

This different impact in the two locations is also reflected in the averaged power production. The power production is shown in Fig 10. As expected due to the differences in total precipitations, the optimal power production in Palma is much lower than in Cordova. In Palma that power shows a slight decrease over time while Cordova shows a small increase



Fig.10. Power production at a hydro plant in Cordova and Palma.
It is these slight trends in the overall optimal production combined with the opposite trends in the intermittency which keep the storage needs fairly constant.

Finally, we compare the efficiency in both locations as we did in the previous section. The results are in Fig. 11. Clearly the efficiency is considerably better in Cordova. As with the solar power, this is due to a combination of the increased amount of hydro available ( more precipitation) and the decreased intermittency in Cordova when compared to Palma.



Fig.11. Ratio of the energy storage needed to the averaged daily energy produced by a hydro plant in Cordova and Palma.

\section{Conclusions}

Resilience and reliability of the power systems are critical to the operation of modern society. As renewable energy sources increase their penetration in the power system the impact of their variability grows. As the magnitude and effects of climate change increase many of the renewable resources (such as solar, hydro, and wind generation) will also be impacted both through the availability of the resources and the variability of those resources. In this paper we have done a preliminary analysis of the effects from climate change observed over the last 60 years for two locations. For the sake of brevity, we only show the impact on precipitation (hydro power) and surface solar radiation (solar power) but similar results are found for wind (wind power). The two locations we investigated, Cordova and Palma, where chosen because they are well separated in both 
distance and climate regions (polar versus subtropical). It was found that even over this limited time the effects of climate change can be seen in some of the characteristics we measured and not in others and there were differences in the impacts at the two locations. At both locations the storage needs increased with time for solar but changed little for hydro. The efficiency (using storage needed per production unit as our measure of efficiency) of the hydropower was much higher in Cordova then Palma but was reversed for solar power with Palma much higher efficiency then Cordova. Both of these are expected due to the nature of the weather at the two locations but this shows that this measure can be used to investigate the appropriate mix of renewable generation make the most sense at various locations. It is worth pointing out that currently there are no hydro plants on the Balearic Island though there was one which operated from 1908 till 1962.

In each of these measures the variability of the resource is an important component of the measure. However, the variability, which we characterized with the intermittency parameter, also has a large impact directly on grid reliability $[11,12]$ as does the degree of distribution of the renewable resource. The intermittency parameter for the precipitation showed a small increase for Palma, a larger increase for Lake Oroville and little change for Cordova. It showed little change for the solar radiation data. Both the change over time of the intermittency parameter and the differences with location are very important for understanding risk (failure risk) for both transmission grids and microgrids as the penetration of the high variability renewable electric power generation sources increases. These metrics form the structure for a framework that can quantify the impacts of climate and climate change on both transmission systems and microgrids. It importantly also points toward how one can mitigate the impacts with energy storage. The next step in this work is to apply these methods to longer time series, more locations and future climate scenarios to quantify the impacts and risk in various locations as we move into the new climate conditions.

\section{Acknowledgements}

The results contain modified Copernicus Climate Change Service information 2021. Neither the European Commission nor ECMWF is responsible for any use that may be made of the Copernicus information or data it contains. Uranus, a supercomputer cluster located at Universidad Carlos III de Madrid (Spain) funded jointly by EU FEDER funds and by the Spanish Government via the National Research Project Nos. UNC313- 4E2361, ENE2009-12213-C03-03, ENE2012-33219, and ENE2012-31753 was used for this project. PC and DG acknowledge financial support from from Ministerio de Ciencia e Innovación (Spain), the Agencia Estatal de Investigación (AEI, Spain), and the Fondo Europeo de Desarrollo Regional (FEDER, EU) under grant PACSS (RTI2018-093732-B-C22) and the Maria de Maeztu program for Units of Excellence in R\&D (MDM-20170711). DN gratefully acknowledges support from DOE Project GMLC 1.5.02 - Resilient Alaskan Distribution system Improvements using Automation, Network analysis, Control, and Energy storage (RADIANCE). USB acknowledges support from the National Science Foundation under award \#OIA-1753748 and by the State of Alaska for material which this work is based upon.

\section{References}

[1] IPCC, Climate Change 2013: The Physical Science Basis. Working Group I Contribution to the Fifth Assessment Report of the Intergovernmental Panel on Climate Change, Cambridge University Press., 2014.

[2] Gernaat, D.E.H.J., H.S. de Boer, V. Daioglou, S.G. Yalew, C. Müller, and D.P. van Vuuren, "Climate change impacts on renewable energy supply", Nature Climate Change 11(2), 2021, pp. 119-125.

[3] Vavrus, S.J., U.S. Bhatt, and V.A. Alexeev, "Factors Influencing Simulated Changes in Future Arctic Cloudiness", Journal of Climate 24(18), 2011, pp. 4817-4830.

[4] Lader, and R.T. et al., "Downscaled projections of hydroclimatic extremes in Southeast Alaska", in prep, 2021.

[5] Pryor, S.C., and R.J. Barthelmie, "Climate change impacts on wind energy: A review", Renewable and Sustainable Energy Reviews 14(1), 2010, pp. 430437.

[6] Bathiany, S., V. Dakos, M. Scheffer, and T.M. Lenton, "Climate models predict increasing temperature variability in poor countries", Science Advances 4(5), 2018, pp. eaar5809.

[7] Pendergrass, A.G., R. Knutti, F. Lehner, C. Deser, and B.M. Sanderson, "Precipitation variability increases in a warmer climate", Scientific Reports 7(1), 2017, pp. 17966.

[8] Vogel, M.M., M. Hauser, and S.I. Seneviratne, "Projected changes in hot, dry and wet extreme events clusters in CMIP6 multi-model ensemble", Environmental Research Letters 15(9), 2020, pp. 094021.

[9] Pes, M.P., E.B. Pereira, J.A. Marengo, F.R. Martins, D. Heinemann, and M. Schmidt, "Climate trends on 
the extreme winds in Brazil", Renewable Energy 109, 2017, pp. 110-120.

[10] Day, J.J., and K.I. Hodges, "Growing Land-Sea Temperature Contrast and the Intensification of Arctic Cyclones", Geophysical Research Letters 45(8), 2018, pp. 3673-3681.

[11] Newman, D.E., B.A. Carreras, M. Kirchner, and I. Dobson, "The Impact of Distributed Generation on Power Transmission Grid Dynamics", 2011 44th Hawaii International Conference on System Sciences 1, 2011, pp. 1-8.

[12] Carreras, B.A., P. Colet, J.M. Reynolds-Barredo, and D. Gomila, "Resilience and performance of the power grid with high penetration of renewable energy sources: the Balearic Islands as a case study", submitted, n.d.

[13] Carreras, B., J.-M. Reynolds-Barredo, D. Newman, and I. Dobson, "The Impact of Incorporating Wind Energy in the Electric Grid", Proceedings of the 53rd Hawaii International Conference on System Sciences, 2020.

[14] Carreras, B.A., J.M. Reynolds-Barredo, and and D.E. Newman, "Optimizing the Impact of Incorporating Wind Energy in the Electric Grid", Journal of Strategic Innovation and Sustainability 15(5), 2020.

[15] information, N.N.C. for E., "Climate at a Glance: Global Time Series", 2021. https://www.ncdc.noaa.gov/cag/

[16] Solaun, K., and E. Cerdá, "Climate change impacts on renewable energy generation. A review of quantitative projections", Renewable and Sustainable Energy Reviews 116, 2019, pp. 109415.

[17] Yalew, S.G., M.T.H. van Vliet, D.E.H.J. Gernaat, et al., "Impacts of climate change on energy systems in global and regional scenarios", Nature Energy 5(10), 2020, pp. 794-802.

[18] Meneveau, C., and and K.R. Sreenivasan, "Simple multifractal cascade model for fully developed turbulence.", Physical review letters 59(13), 1987, pp. 1424.

[19] Meneveau, C., and K. Sreenivasan, "The multifractal nature of turbulent energy dissipation", Journal of Fluid Mechanics 224, 1991, pp. 429484.

[20] Carreras, B.A., V.E. Lynch, D.E. Newman, et al., "Intermittency of plasma edge fluctuation data: Multifractal analysis", Physics of Plasmas 7(8), 2000, pp. 3278-3287.

[21] Hentschel, H.G. E., and and I. Procaccia, "The infinite number of generalized dimensions of fractals and strange attractors.", Physica D: Nonlinear Phenomena 8(3), 1983, pp. 435-444.

[22] Hersbach, H., Bell, et al., "The ERA5 global reanalysis", Quarterly Journal of the Royal Meteorological 146(730), 2020, pp. 1999-2049.

[23] Bekryaev, R.V., I.V. Polyakov, and V.A. Alexeev, "Role of Polar Amplification in Long-Term Surface Air Temperature Variations and Modern Arctic Warming", Journal of Climate 23(14), 2010, pp. 3888-3906.

[24] “AEMET", n.d. http://www.aemet.es/en/portada

[25] Zakeri, G., "Potential Impact of Climate Change on the New Zealand Electricity Market", Proceedings of the 53rd Hawaii International Conference on System Sciences, 2020.

[26] Swain, D.L., B. Langenbrunner, J.D. Neelin, and A. Hall, "Increasing precipitation volatility in twenty-first-century California", Nature Climate Change 8(5), 2018, pp. 427-433. 